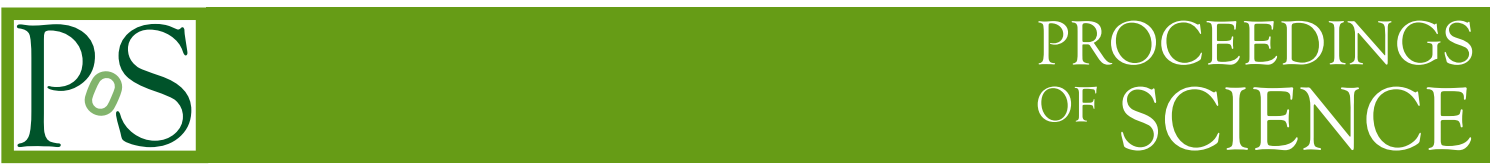

\title{
Precision timing for the High Luminosity Upgrade of CMS
}

\author{
Rachel Yohay*t \\ Florida State University (US) \\ E-mail: rachel.yohay@cern.ch
}

\begin{abstract}
The projected proton beam intensity of the High Luminosity Large Hadron Collider (HL-LHC), slated to begin operation in 2026, will result in between 140 and 200 concurrent proton-proton interactions per $25 \mathrm{~ns}$ bunch crossing. The scientific program of the HL-LHC, which includes precision Higgs coupling measurements, measurements of vector boson scattering, and searches for new heavy or exotic particles, will benefit greatly from the enormous HL-LHC dataset. However, particle reconstruction and correct assignment to primary interaction vertices presents a formidable challenge to the LHC detectors that must be overcome in order to reap that benefit. Time tagging of minimum ionizing particles (MIPs) produced in LHC collisions with a resolution of $30 \mathrm{ps}$ provides further discrimination of interaction vertices in the same $25 \mathrm{~ns}$ bunch crossing beyond spatial tracking algorithms. The Compact Muon Solenoid (CMS) Collaboration is pursuing two technologies to provide MIP time tagging for the HL-LHC detector upgrade: LYSO:Ce crystals read out by silicon photomultipliers (SiPMs) for low radiation areas and silicon low gain avalanche detectors (LGADs) for high radiation areas. This talk will motivate the need for a dedicated timing layer in the CMS upgrade, describe the two technologies and their performance, and present simulations showing the improvements in reconstructed observables afforded by four dimensional tracking.
\end{abstract}

The 26th International Workshop on Vertex Detectors

10-15 September, 2017

Las Caldas, Asturias, Spain

\footnotetext{
* Speaker.

${ }^{\dagger}$ On behalf of the CMS Collaboration.
} 


\section{Introduction}

Slated to begin operation in 2026, the HL-LHC will achieve instantaneous proton-proton luminosities of 5-7 $\times 10^{34} \mathrm{~cm}^{-2} \mathrm{~s}^{-1}$, corresponding to a peak of 140-200 pp interactions per $25 \mathrm{~ns}$ bunch crossing, or pileup. At this brightness, it is expected that $3000-4000 \mathrm{fb}^{-1}$ will be integrated within a ten-year run period, yielding an HL-LHC dataset approximately one order of magnitude larger than the projected final LHC dataset. This is a requirement of the HL-LHC physics program, which includes precision measurements of Higgs couplings, searches for rare processes predicted by extensions of the standard model (SM), and studies of rare SM processes. In particular, an integrated luminosity of $3000 \mathrm{fb}^{-1}$ is required to constrain uncertainties on the top-Higgs coupling to better than $10 \%$, and in some cases ( $\mathrm{ZH}$ and $\mathrm{WH}$, for example) is sufficient to limit coupling uncertainties to better than $5 \%$ [1].

Significant upgrades to the CMS detector [2] are necessary to be able to exploit the increased HL-LHC instantaneous and integrated luminosity. In its current form, the CMS Phase 2 upgrade plan for the HL-LHC [3] includes

- a full replacement of the silicon charged particle tracker, with a new design that is radiation tolerant till 2036, incorporates an online track trigger system, and extends coverage to $|\eta|<$ 4.0 ;

- a full replacement of the endcap $(1.5<|\eta|<3.0)$ calorimeter, with a new design that is radiation tolerant till 2036 and ideally suited to particle flow reconstruction [4]; and

- a replacement of the front end readout electronics, back end data acquisition (DAQ) system, and back end trigger system to be be compatible with a $750 \mathrm{kHz}$ trigger rate and up to 200 pileup interactions per bunch crossing.

The design goal of the CMS Phase 2 upgrades is to maintain similar performance with 200 pileup interactions as the current detector with 50 pileup interactions. Although the current Phase 2 design makes significant progress toward achieving that goal, the effects of high pileup can be more thoroughly mitigated with the addition of a timing detector for minimum ionizing particles (MIPs) that achieves $30 \mathrm{ps}$ timing resolution. Section 2 of this article describes some of the problems caused by high pileup that can be solved with MIP timing information. In Section 3, the design for a CMS MIP timing detector to accompany the Phase 2 upgrades is presented. Finally, the projected performance of this detector is given in Section 4, and concluding remarks follow in Section 5.

\section{Effects of high pileup}

With 200 pileup interactions per $25 \mathrm{~ns}$ bunch crossing, separate interaction vertices that occur at the same $\mathrm{z}$-coordinate, where the $\mathrm{z}$ axis is parallel to the beam line, can get merged into a single reconstructed vertex. Figure 1 illustrates this phenomenon in simulated $\mathrm{H} \rightarrow \gamma \gamma$ events, where $\mathrm{H}$ is the SM Higgs boson. The red dots in the figure indicate the z-coordinate and time coordinate of simulated interaction vertices. The black crosses indicate the locations of reconstructed tracks using both spatial and time information ("4D tracks"), where the vertex z-coordinate resolution is 10-100 
$\mu \mathrm{m}$ [5] and a track timing resolution of $30 \mathrm{ps}$ is assumed, while the blue open circles indicate the locations of vertices ("4D vertices") reconstructed using those 4D tracks. In the case of 50 pileup interactions, shown in Fig. 1 (left), the z-coordinate resolution is sufficient to separate the vertices. However, in the case of 200 pileup interactions, shown in Fig. 1 (right), the density of tracks is sufficiently high that two or more vertices may be merged into one inaccurately reconstructed vertex using spatial information alone ("3D vertices"), as indicated by the vertical dashed yellow lines. Vertex merging can be seen when multiple red dots (simulated vertices) fall on the same vertical dashed yellow line (reconstructed 3D vertices). Fig. 1 is meant only to illustrate the vertex merging phenomenon - the thickness of the vertical dashed yellow lines is greater than the expected $10-100 \mu \mathrm{m}$ vertex z-coordinate resolution. However, in events with 200 pileup interactions, the expected merging rates are $15 \%$ with $3 \mathrm{D}$ vertexing, but less than $2 \%$ with $4 \mathrm{D}$ vertexing.
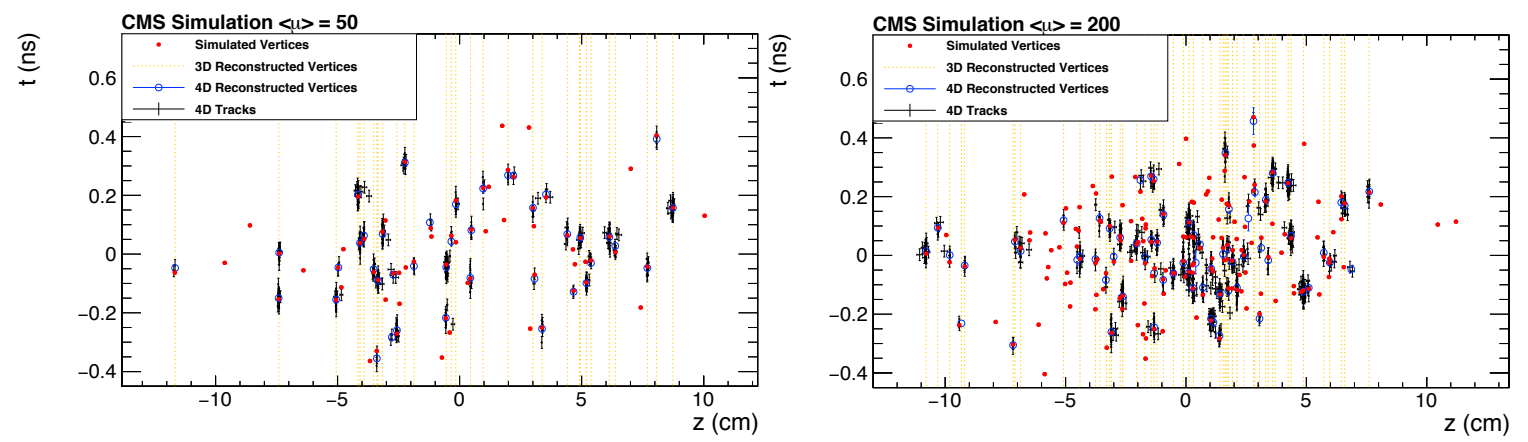

Figure 1: The distribution, versus z-coordinate and time coordinate, of simulated interaction vertices (red), vertices reconstructed using spatial information only (vertical dashed yellow lines), vertices reconstructed using spatial and time information (open blue circles), and tracks reconstructed using spatial and time information (black crosses). $\mathrm{z}=\mathrm{t}=0$ corresponds to the centre of the true LHC beam crossing position. An example of two vertices merged in 3D reconstruction but not in 4D reconstruction can be seen at $\mathrm{z} \sim-7.5$ $\mathrm{cm}$ in the left plot. Left: 50 pileup interactions per crossing. Right: 200 pileup interactions per crossing.

Vertex merging affects physics performance in a variety of ways. Because tracks are associated to the wrong primary or secondary vertices, jet identification and energy resolution, as well as missing transverse energy $\left(\mathrm{E}_{\mathrm{T}}^{\mathrm{miss}}\right.$ ) resolution, suffer. Furthermore, as track momentum is used in calculating isolation energy sums around a candidate photon or charged lepton, merging can reduce the discriminating power of isolation for these objects. In hard scatter events with no prompt tracks, such as $\mathrm{H} \rightarrow \gamma \gamma$ events, the vertex with the largest track $\sqrt{\sum p_{T}^{2}}$ is reconstructed as the corresponding interaction vertex. Vertex merging increases the number of tracks associated to a given reconstructed vertex, which makes a typical low- $\sqrt{\sum p_{\mathrm{T}}^{2}}$ pileup vertex look more like a high$\sqrt{\sum p_{\mathrm{T}}^{2}}$ hard scatter vertex. This in turn leads to a higher frequency of incorrect primary vertex assignments, thereby degrading diphoton mass resolution.

The current CMS Phase 2 proposal only partially mitigates the problem of vertex merging with a design feature of 30 ps time resolution on high energy electromagnetic showers, such as those resulting from $\mathrm{H} \rightarrow \gamma \gamma$, deduced from calorimeter time reconstruction. With this resolution, the vertex z-coordinate can be triangulated from the time coordinates of the two photon showers, as long as they are well separated in pseudorapidity $\eta$. This is illustrated in Figure 2. As the difference in $\eta$ between the two photon showers decreases, a larger fraction of $\mathrm{H} \rightarrow \gamma \gamma$ vertices 
cannot be reconstructed within $1 \mathrm{~cm}$ of the true vertex. In these cases, a more precise vertex hypothesis from 4D MIP tracks is needed to break the degeneracy of consistent vertex hypotheses coming from calorimeter shower timing alone.
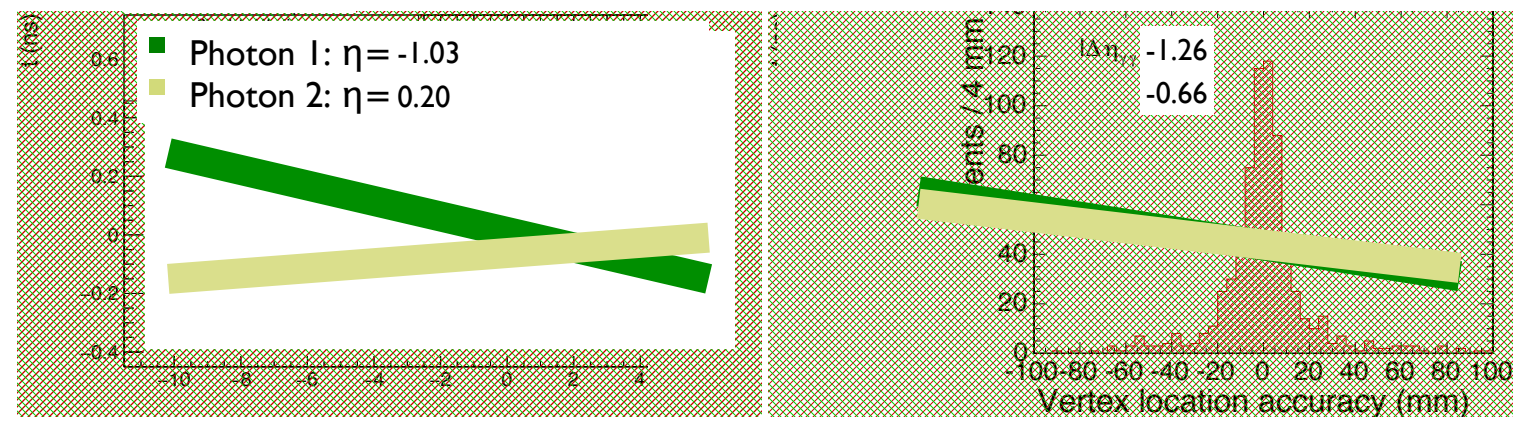

Figure 2: Depictions of the $\mathrm{H} \rightarrow \gamma \gamma$ vertex triangulation method using the space of all vertex hypotheses consistent with the time difference measured between the two photons when the difference in $\eta$ between the two photon showers is greater than (left) and less than (right) 0.8 . The spread in z-positions compatible with the overlap between the two photon hypotheses gives a measure of the spread in vertex location accuracy.

More effective pileup rejection is needed in order to meet the CMS Phase 2 design goal of maintaining similar performance with 200 pileup interactions as the current detector with 50 pileup interactions. Precision timing of MIPs adds a crucial independent handle on pileup identification. With 30 ps MIP timing resolution, the merged vertex rate at the HL-LHC is reduced to the current level of a few percent. As shown in Fig. 1 (right), 4D tracking recovers a high efficiency for accurately reconstructing vertices in the spacetime plane. From this improvement, the performance for $\mathrm{H} \rightarrow \gamma \gamma$ reconstruction, lepton identification using isolation, and jet and $\mathrm{E}_{\mathrm{T}}^{\mathrm{miss}}$ energy resolution are similarly preserved.

\section{Precision timing detector proposal}

A proposal has recently been made to the LHC Experiments Committee (LHCC) to add a MIP timing detector to the CMS Phase 2 upgrade plan. The CMS MIP Timing Detector (MTD) would cover $|\eta|<3.0$, as does the calorimeter, and would maintain a time resolution below $50 \mathrm{ps}$ throughout the HL-LHC run period. The MTD proposal consists of a barrel (Barrel Timing Layer, or BTL) section and an endcap (Endcap Timing Layer, or ETL) section, each using technologies tailored to specific radiation hardness needs. In the relatively low-dose, low-fluence region of the BTL, LYSO:Ce scintillating crystals are read out by silicon photomultipliers (SiPMs). In the ETL, where fluences of up to $10^{15} \mathrm{n}_{\mathrm{eq}} / \mathrm{cm}^{2}$ are expected, ${ }^{1}$ more radiation tolerant silicon low gain avalanche detectors (LGADs) [7, 8] are employed. An r-z view of the inner layers of the CMS Phase 2 detector highlighting the location of the MTD is shown in Figure 3.

\subsection{Barrel Timing Layer (BTL)}

The proposed BTL covers $|\eta|<1.5$, a favored region for high- $p_{\mathrm{T}}$ physics signals such as $\mathrm{H} \rightarrow \gamma \gamma$. Improved vertexing in this region provides significant gains in the reconstruction of final states with neutral particles.

\footnotetext{
${ }^{1} 1 \mathrm{n}_{\mathrm{eq}}=11-\mathrm{MeV}$ neutron (see Ref. [6] for details).
} 


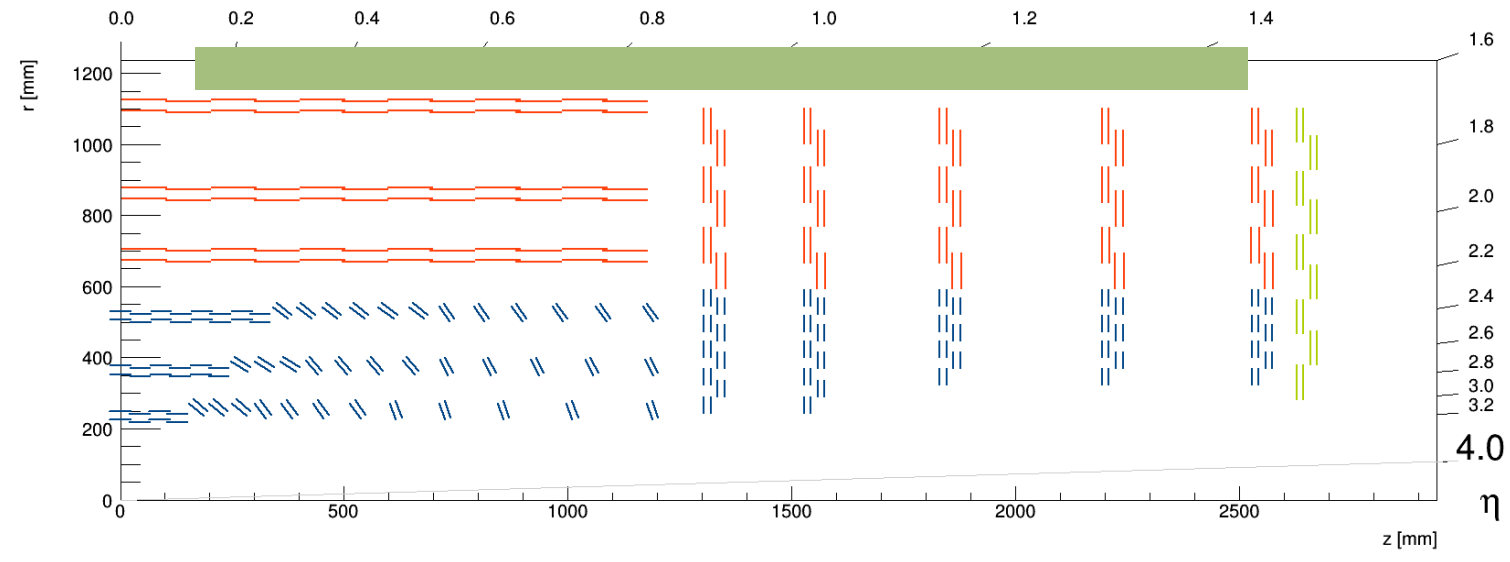

Figure 3: $r-z$ view of the inner layers of the CMS Phase 2 detector. The red and blue lines indicate the location of CMS Outer Tracker modules. The green lines indicate the location of the the proposed BTL and ETL.

The BTL consists of a layer of LYSO:Ce crystal tiles, each of $12 \times 12 \mathrm{~mm}^{2}$ area, read out by a SiPM of nominal $4 \times 4 \mathrm{~mm}^{2}$ area glued to the center of the tile. The tile thicknesses range from $2.40-3.75 \mathrm{~mm}$, depending on pseudorapidity, to maintain a uniform material budget. Cracks between tiles are minimized with a $0.5 \mathrm{~mm}$ edge overlap. With this geometry, the predicted occupancy in events with 200 pileup interactions is $<3 \%$ assuming a 0.5 MIP threshold per channel (1 $\mathrm{MIP} \sim 5000$ photoelectrons). To mitigate increasing SiPM dark current with radiation damage, the entire BTL will be enclosed in a sealed, dry volume cooled to $-30^{\circ} \mathrm{C}$ with $\mathrm{CO}_{2}$ [9].

The LYSO:Ce + SiPM time resolution has been measured for different tile shapes in a $150 \mathrm{GeV}$ muon test beam at CERN [10]. After measuring the muon arrival time difference (coincidence time resolution, or CTR) between two LYSO:Ce + SiPM tiles, the single-tile time resolution is estimated as CTR $/ \sqrt{2}$. Figure 4 shows the measured CTR distribution, before and after timewalk ${ }^{2}$ correction, for two different crystal areas. For both geometries, the timewalk-corrected single-tile time resolution is better than $25 \mathrm{ps}$.

After 10 years of HL-LHC operation, the BTL is expected to integrate a fluence of 1.3-1.6 $\times 10^{14} \mathrm{n}_{\mathrm{eq}} / \mathrm{cm}^{2}$ and total ionizing dose of $20 \mathrm{kGy}$. The radiation-induced absorption coefficient (RIAC) for LYSO tiles, after an irradiation fluence of $2 \times 10^{14}$ protons per square centimeter in an $800 \mathrm{MeV}$ beam at Los Alamos, has been measured to be $\sim 3 \mathrm{~m}^{-1}$ [11], a sufficiently low value for $\sim 3 \mathrm{~mm}$ thick BTL tiles. After gamma irradiation up to $100 \mathrm{kGy}$, the measured relative light loss in LYSO crystals was $\sim 10 \%$ [12]. The combined RIAC and light loss measurements indicate that LYSO tiles should survive the expected HL-LHC radiation damage.

The remaining technological hurdles to a fully radiation tolerant design concern the SiPM photodetectors. Figure 5 shows different aging simulations for a $12 \times 12 \mathrm{~mm}^{2}$ crystal, in terms of single-tile time resolution vs. SiPM dark count rate (DCR), depending on SiPM photon detection efficiency (PDE). The solid curves in Fig. 5 are pure simulation, while the points indicate extrapolations of measurements made under different conditions (in the context of the CMS Hadronic

\footnotetext{
${ }^{2}$ When a current pulse is read out by a threshold discriminator, the term "timewalk" describes the effect whereby the measured pulse time depends on the pulse amplitude. The pulse time can be corrected if the pulse amplitude is known.
} 

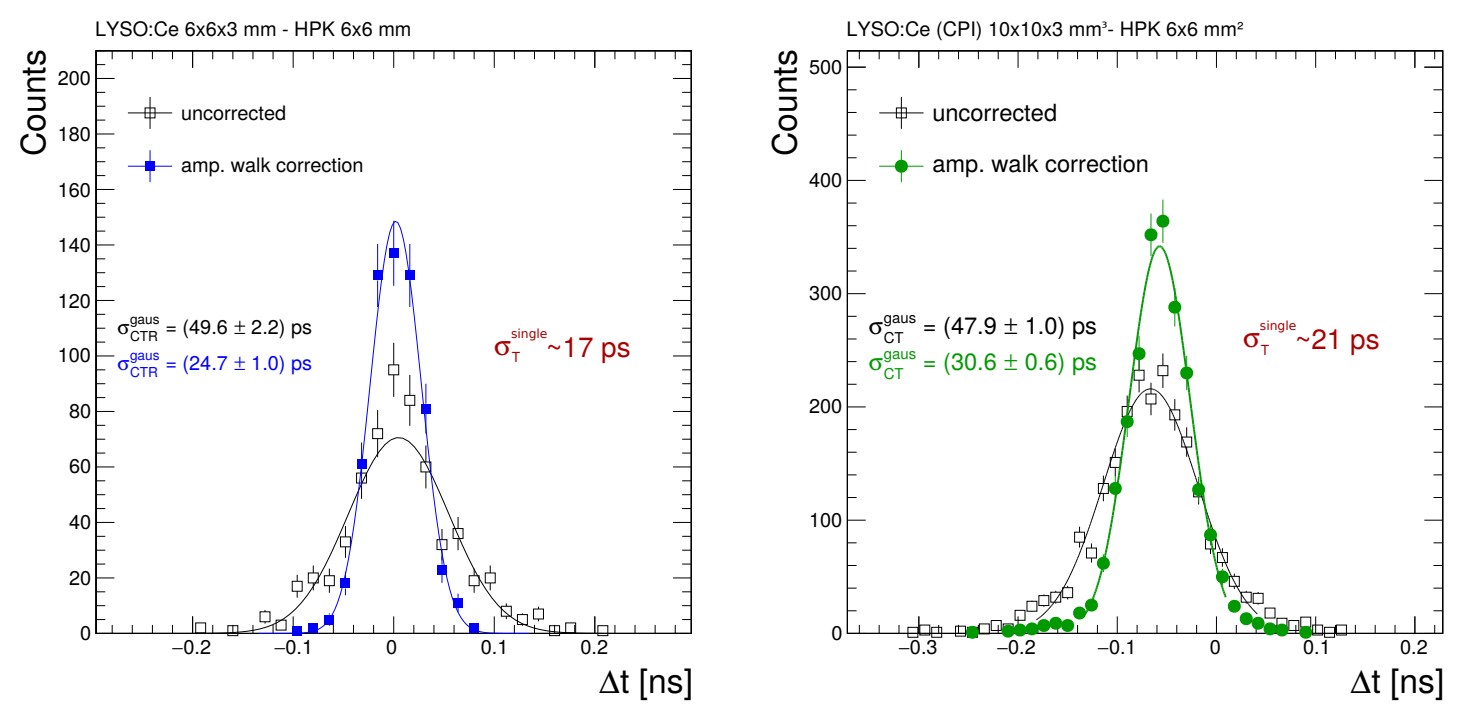

Figure 4: CTR distributions measured in a $150 \mathrm{GeV}$ muon test beam at CERN, before (open markers) and after (filled markers) timewalk correction, for $6 \times 6 \mathrm{~mm}^{2}$ crystal tiles (left) and $10 \times 10 \mathrm{~mm}^{2}$ crystal tiles (right). Reprinted from Ref. [10].

Calorimeter Phase 1 upgrade; cf. Refs. $[13,14,15])$ to the expected conditions of the BTL at its end of life: either 1.5 or $2.0 \mathrm{~V} \mathrm{SiPM}$ bias overvoltage, as indicated in the labels, and $2 \times 10^{14}$ $\mathrm{n}_{\mathrm{eq}} / \mathrm{cm}^{2}$ integrated fluence. The operating temperature assumed in the extrapolations is the design temperature of $-30^{\circ} \mathrm{C}$. These simulations and extrapolations help to guide the development of specifications for potential SiPM vendors, with the goal of maintaining the time resolution at end of life below $40 \mathrm{ps}$. In addition to increasing the radiation hardness of SiPMs, it is possible to compensate SiPM performance with increased light collection (via reflective tile wrappings or thicker tiles) or reduced SiPM area (and therefore reduced DCR).

\subsection{Endcap Timing Layer (ETL)}

The ETL covers $1.5<|\eta|<3.0$, where 3D tracking is more difficult with respect to the central barrel region. Silicon LGADs are used as sensors. Through a combination of small thickness (50 $\mu \mathrm{m}$ ) to reduce charge collection time and an extra doping layer to generate moderate (factor $\sim 20$ ) gain, these LGADs are engineered to have a high slew rate and therefore good time resolution. Each sensor has an area of $48 \times 96 \mathrm{~mm}^{2}$, with an individual pixel size of $1 \times 3 \mathrm{~mm}^{2}$. This geometry insures an occupancy $<5 \%$ and per-pixel capacitance of $7 \mathrm{pF}$ in HL-LHC conditions. As for the BTL, the ETL is cooled to $-30^{\circ} \mathrm{C}$.

The LGAD time resolution has been measured for different reverse bias voltages in a 180 $\mathrm{GeV}$ pion test beam at CERN [16]. Figure 6 shows the measured CTR distributions, where the time difference is measured between an LGAD and a SiPM. For bias voltages above $200 \mathrm{~V}$, singleLGAD time resolutions of better than 35 ps have been observed.

After 10 years of HL-LHC operation, the largest fluence expected at the ETL is $\sim 1 \times 10^{15}$ $\mathrm{n}_{\mathrm{eq}} / \mathrm{cm}^{2}$. At present, the time resolution of LGAD devices under consideration for the ETL exceeds the design goal of $50 \mathrm{ps}$ at this fluence by approximately $10 \mathrm{ps}$, as shown in Figure 7. The effect 


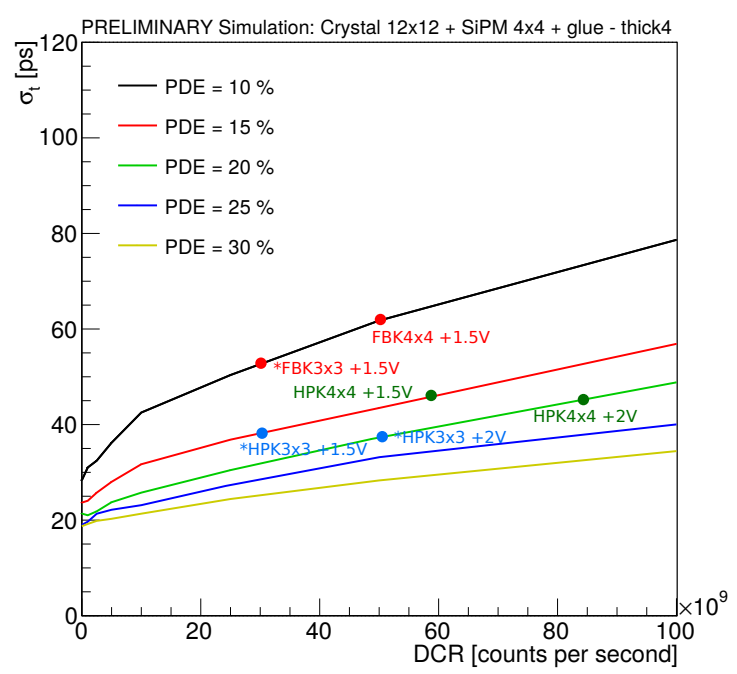

Figure 5: Different aging simulations for a $12 \times 12 \mathrm{~mm}^{2}$ crystal, in terms of single-tile time resolution vs. SiPM DCR, depending on SiPM PDE. The solid curves are pure simulation, while the points indicate extrapolations of measurements made under different conditions (in the context of the CMS Hadronic Calorimeter Phase 1 upgrade; cf. Refs. $[13,14,15])$ to the expected conditions of the BTL at its end of life: either 1.5 or $2.0 \mathrm{~V} \mathrm{SiPM}$ bias overvoltage, as indicated in the labels, and $2 \times 10^{14} \mathrm{n}_{\mathrm{eq}} / \mathrm{cm}^{2}$ integrated fluence. The operating temperature assumed in the extrapolations is the design temperature of $-30^{\circ} \mathrm{C}$.

of radiation damage is to decrease the gain of the LGADs, which decreases the slew rate and worsens time resolution. However, Fig. 7 illustrates that timing performance can be recovered with increased bias voltage [17]. Two avenues are consequently being explored in the development of a sufficiently radiation hard LGAD: engineering the gain layer to better withstand radiation damage, and developing thin sensors that can withstand high external bias voltages.

\section{Performance}

Simulations show that the performance of the CMS Phase 2 detector is significantly improved when high resolution timing information, like that provided by the MTD described in Sec. 3, is available. As argued in Sec. 2, calorimeter timing of high energy photon showers alone is insufficient to triangulate the position of the $\mathrm{H} \rightarrow \gamma \gamma$ vertex when the two photons are close in pseudorapidity. Figure 8 (left) illustrates how correlation with precise timing information can reduce the ambiguity in choosing the correct vertex. The plot shows the z-t distribution of simulated, 3D, and $4 \mathrm{D}$ vertices as in Fig. 1, with the space of vertex hypotheses from calorimeter-only timing of the two photons from Fig. 2 (right) overlaid. (Only 20 pileup interactions per event are simulated to aid in the illustration, but vertex location accuracy is also improved for 200 interactions.) Correlation of the vertex hypotheses consistent with both photon arrival times and the 4D MIP tracking leads to the identification of the correct vertex at $\mathrm{z} \sim-2 \mathrm{~cm}$. As shown in Figure 8 (right), better vertex location accuracy translates to better diphoton mass resolution (red curve vs. blue curve). This example highlights the importance of the BTL in the kinematic reconstruction of decays of heavy particles to neutral particles. 

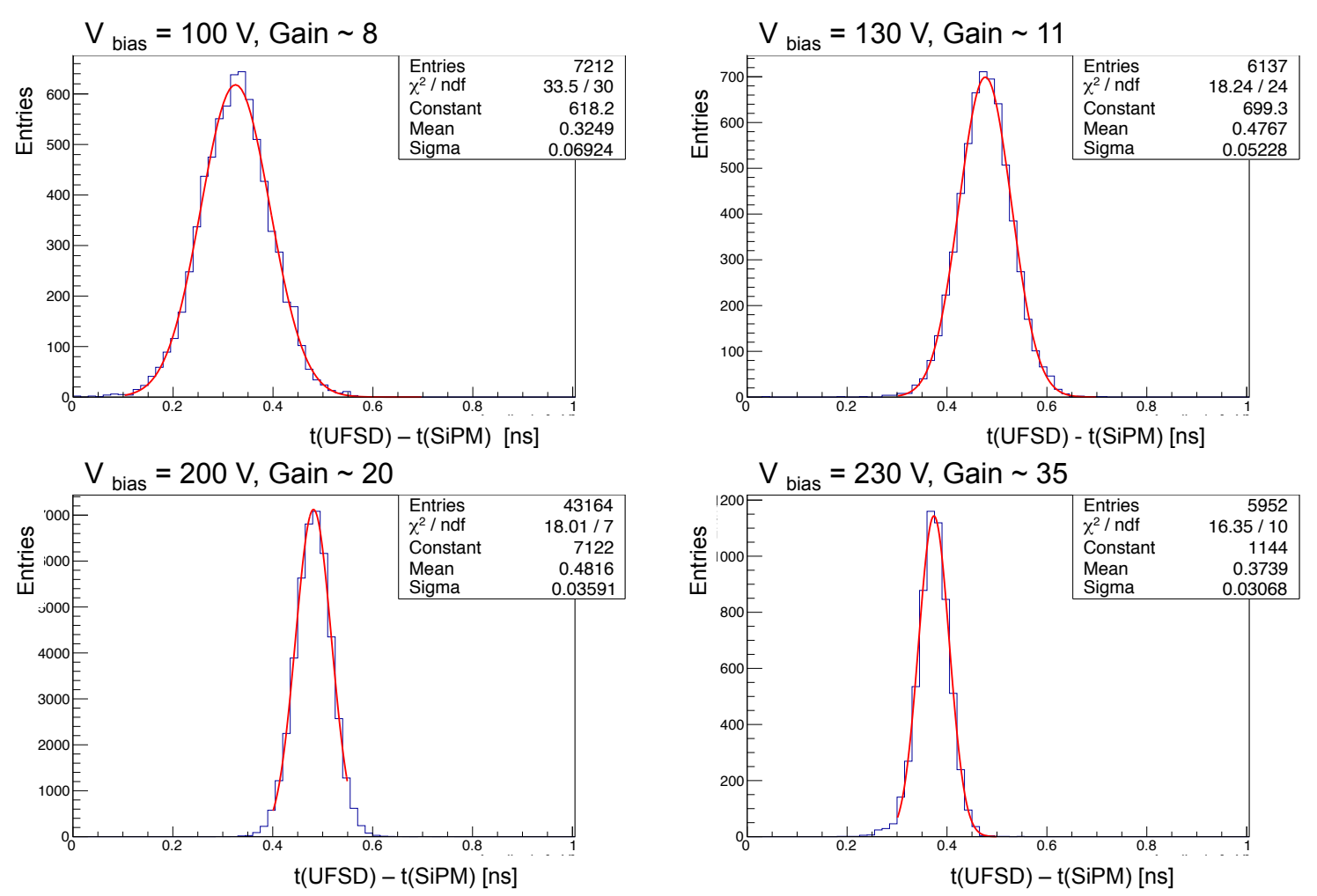

Figure 6: LGAD (labeled "UFSD", or Ultra-Fast Silicon Detector, another term for LGAD) CTR distributions measured in a $180 \mathrm{GeV}$ pion beam at CERN for reverse bias voltages of $100 \mathrm{~V}$ (top left), $130 \mathrm{~V}$ (top right), $200 \mathrm{~V}$ (bottom left), and $230 \mathrm{~V}$ (bottom right). The red curves show the results of a Gaussian fit. Reprinted from Ref. [16].

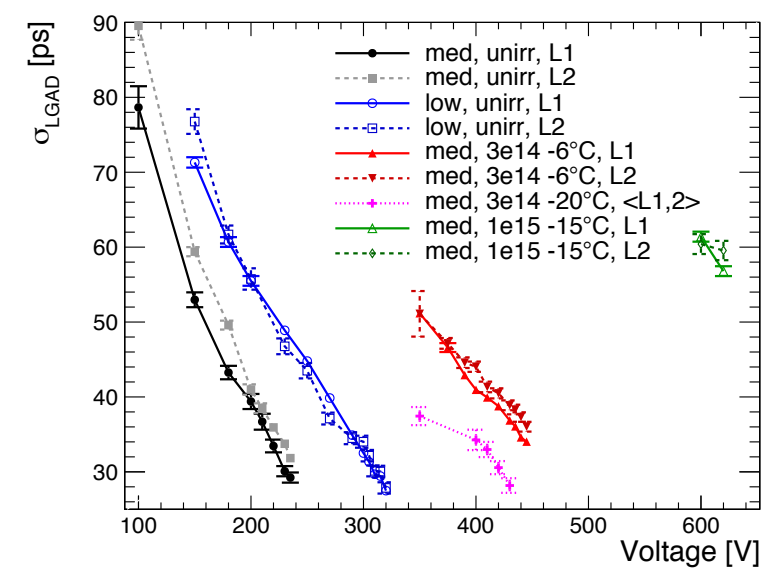

Figure 7: LGAD time resolution vs. bias voltage for devices irradiated to fluences up to $10^{15} \mathrm{n}_{\mathrm{eq}} / \mathrm{cm}^{2}$. Reprinted from Ref. [17]. 

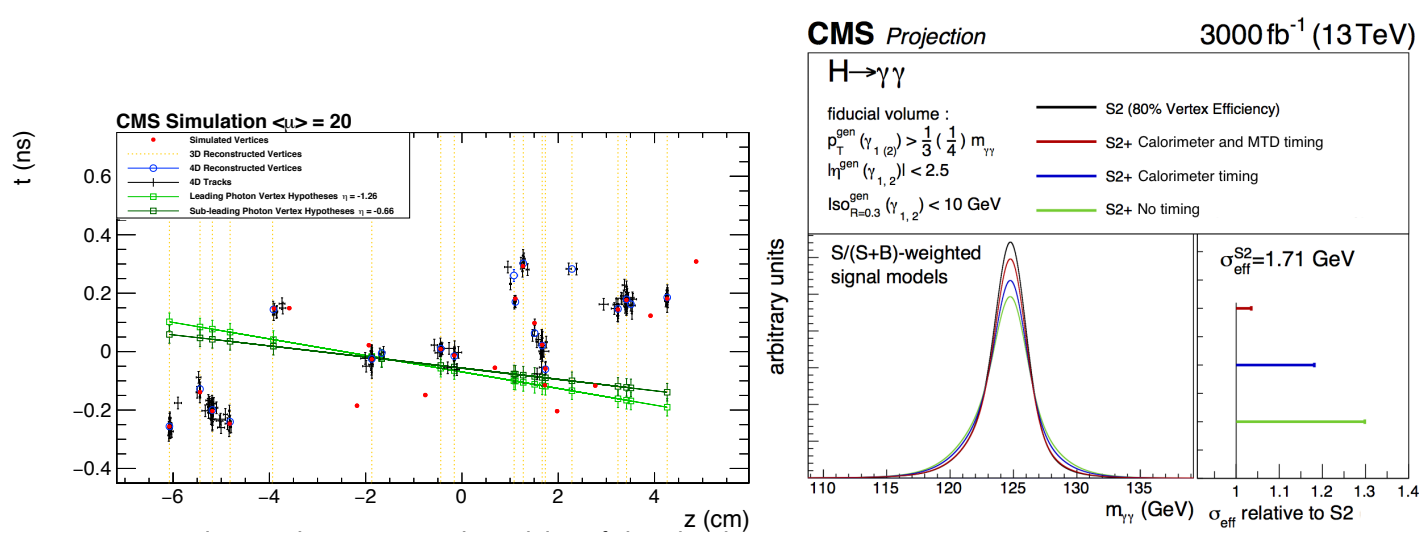

Figure 8: Left: $z$-t distribution of simulated, 3D, and 4D vertices as in Fig. 1, with the space of vertex hypotheses from calorimeter-only timing of the two photons from Fig. 2 (right) overlaid in green. Only 20 pileup interactions per event are simulated to aid in the illustration. Right: $\mathrm{H} \rightarrow \gamma \gamma$ diphoton mass resolution as measured in LHC Run 2 ("S2", black), projected for 140 pileup interactions ("S2+") with no timing reconstruction at all (green), projected for 140 pileup interactions with calorimeter timing only (blue), and projected for 140 pileup interactions with both calorimeter and MTD timing (red).

Isolation as a discriminating variable between prompt charged leptons and jets loses its power when additional pileup tracks are mis-assigned to the lepton primary vertex. Figure 9 shows that with precision timing information, mis-assignment is reduced to $~$ LHC levels in HL-LHC conditions. As expected, isolation efficiencies for charged leptons are also recovered to near LHC levels when requiring $3 \sigma$ compatibility between isolation track time and lepton vertex time. This is illustrated in Figure 10 for muons and hadronic tau decays.

$\mathrm{E}_{\mathrm{T}}^{\mathrm{miss}}$ reconstruction, which relies on accurate knowledge of the 4-vectors of all particles in the event, is degraded in HL-LHC conditions due to vertex merging and confusion. The availability of precision time information significantly limits that degradation. Figure 11 demonstrates that there is some reduction in the tails of the $\mathrm{E}_{\mathrm{T}}^{\text {miss }}$ distribution to be expected from the inclusion of timing information afforded by the MTD in the reconstruction.

\section{Conclusion}

To exploit the HL-LHC physics potential, significant upgrades to the tracker, calorimeter, and electronics of the CMS detector are required. However, these planned upgrades do not fully address the problems caused by vertex merging in events with 140-200 pileup interactions per crossing: degraded vertex location accuracy for processes with neutral particles in the final state, such as $\mathrm{H} \rightarrow \gamma \gamma$; reduced discriminating power of charged lepton isolation due to track-vertex misassignment; and worsened jet energy and $\mathrm{E}_{\mathrm{T}}^{\mathrm{miss}}$ resolution due to the same. A MIP timing detector with better than 50 ps time resolution throughout the HL-LHC run period can provide a crucial piece of disambiguating information, allowing for near-LHC performance to be achieved in HLLHC conditions. The technology to build such a detector within the framework of the CMS Phase 2 upgrades is within reach. 


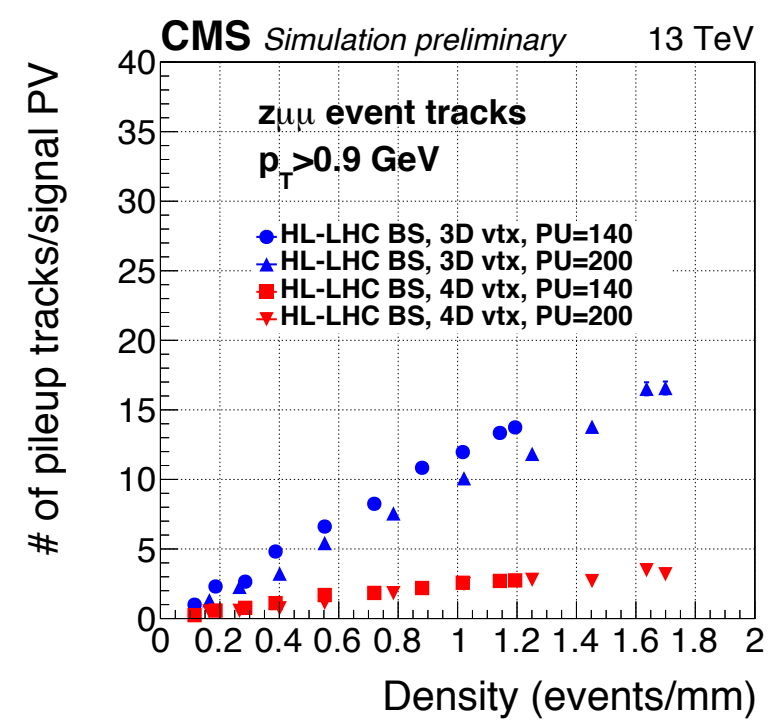

Figure 9: Average number of pileup tracks mis-assigned to the $\mathrm{Z} \rightarrow \mu \mu$ decay vertex vs. interaction density along the z-coordinate (a measure of pileup) for 3D (blue circles and right-side-up triangles) and 4D (red squares and upside down triangles) vertexing. 25 ps vertex timing resolution is assumed. LHC pileup conditions (20 interactions per crossing) correspond to 0.1-0.4 events/mm, HL-LHC minimal pileup conditions (140 interactions per crossing) correspond to 0.4-1.4 events $/ \mathrm{mm}$, and HL-LHC nominal pileup conditions (200 interactions per crossing) correspond to 1.4-1.9 events $/ \mathrm{mm}$. The LHC beamspot size is different in the simulations of 140 and 200 interactions per crossing, which accounts for the small differences between the two curves showing $3 \mathrm{D}$ vertexing.
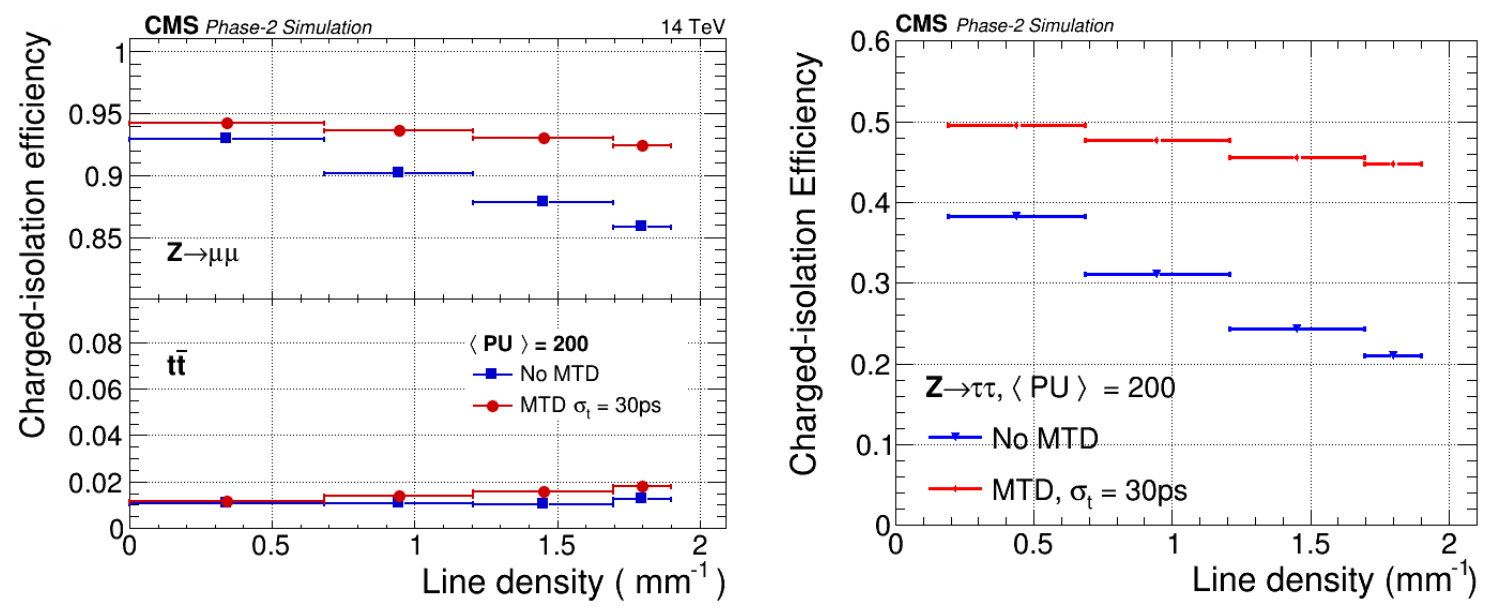

Figure 10: Track isolation efficiency vs. interaction density along the $\mathrm{z}$-coordinate for prompt muons from $\mathrm{Z} \rightarrow \mu \mu$ events and fake muons from $\mathrm{t} \overline{\mathrm{t}}$ events (left) and hadronic tau decays from $\mathrm{Z} \rightarrow \tau \tau$ events (right). A requirement of $3 \sigma$ compatibility between isolation track time and lepton vertex time is imposed. The red (blue) curves represent measurements made with (without) the MTD simulated. 


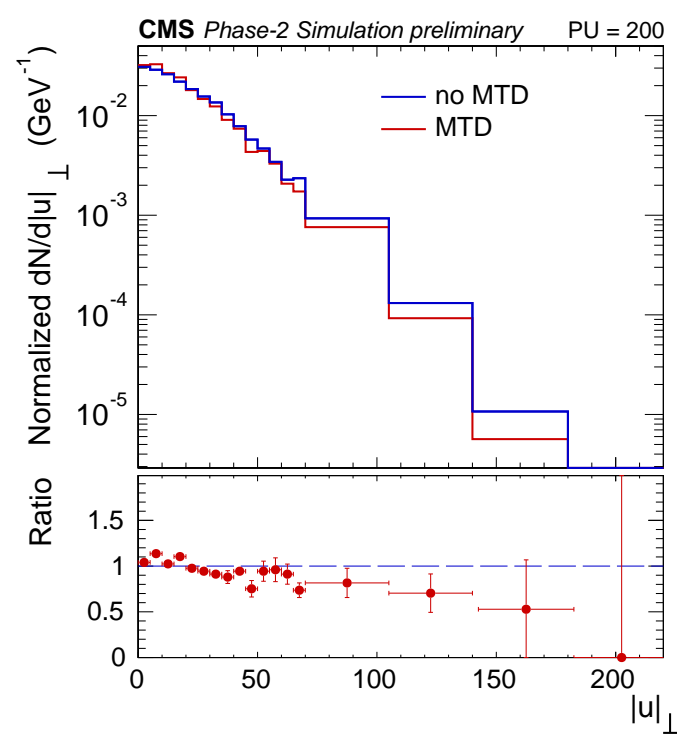

Figure 11: $\mathrm{E}_{\mathrm{T}}^{\mathrm{miss}}$ distribution (normalized to unity) for events with 200 simulated pileup interactions, when $\mathrm{E}_{\mathrm{T}}^{\text {miss }}$ is reconstructed with (red) and without (blue) MTD timing information.

\section{References}

[1] CMS Collaboration, Projected Performance of an Upgraded CMS Detector at the LHC and HL-LHC: Contribution to the Snowmass Process, in Proceedings, 2013 Community Summer Study on the Future of U.S. Particle Physics: Snowmass on the Mississippi (CSS2013): Minneapolis, MN, USA, July 29-August 6, 2013, 2013, 1307 . 7135, https://inspirehep.net/record/1244669/files/arXiv:1307.7135.pdf.

[2] CMS Collaboration, S. Chatrchyan et al., The CMS Experiment at the CERN LHC, JINST 3 (2008) S08004.

[3] D. Contardo, M. Klute, J. Mans, L. Silvestris and J. Butler, Technical Proposal for the Phase-II Upgrade of the CMS Detector, Tech. Rep. CERN-LHCC-2015-010, LHCC-P-008, CMS-TDR-15-02, CERN, Geneva, June, 2015.

[4] CMS Collaboration, A. M. Sirunyan et al., Particle-flow reconstruction and global event description with the CMS detector, JINST 12 (2017) P10003, [1706.04965].

[5] CMS Collaboration, The Phase-2 Upgrade of the CMS Tracker, Tech. Rep. CERN-LHCC-2017-009. CMS-TDR-014, CERN, Geneva, Jun, 2017.

[6] ROSE Collaboration, Fluence normalization based on the NIEL scaling hypothesis, in 3rd ROSE Workshop on Radiation Hardening of Silicon Detectors, DESY Hamburg 12-14 February 1998, 1998.

[7] G. Pellegrini et al., Technology developments and first measurements of Low Gain Avalanche Detectors (LGAD) for high energy physics applications, NIM A765 (2014) 12-16.

[8] N. Cartiglia, R. Arcidiacono, M. Baselga, R. Bellan, M. Boscardin, F. Cenna et al., Design optimization of ultra-fast silicon detectors, NIM A796 (2015) 141-148.

[9] P. Tropea, J. Daguin, P. Petagna, H. Postema, B. Verlaat and L. Zwalinski, $\mathrm{CO}_{2}$ evaporative cooling: The future for tracking detector thermal management, NIM A824 (2016) 473-475. 
[10] M. Lucchini, S. Gundacker, P. Lecoq, A. Benaglia, M. Nikl, K. Kamada et al., Timing capabilities of garnet crystals for detection of high energy charged particles, NIM A852 (2017) 1-9.

[11] F. Yang, L. Zhang, R.-Y. Zhu, J. Kapustinsky, R. Nelson and Z. Wang, Proton induced radiation damage in fast crystal scintillators, NIM A824 (2016) 726-728.

[12] R. Mao, L. Zhang and R.-Y. Zhu, LSO/LYSO crystals for future HEP experiments, J. Phys.: Conf. Ser. 293 (2011) 012004.

[13] Y. Musienko, A. Heering, R. Ruchti, M. Wayne, A. Karneyeu and V. Postoev, Radiation damage studies of silicon photomultipliers for the CMS HCAL phase I upgrade, NIM A787 (2015) 319-322.

[14] A. Heering, Y. Musienko, R. Ruchti, M. Wayne, A. Karneyeu and V. Postoev, Effects of very high radiation on SiPMs, NIM A824 (2016) 111-114.

[15] Y. Musienko, A. Heering, M. Wayne, R. Ruchti, A. Karneyeu and V. Postoev, Studies of SiPMs for the CMS HCAL Upgrade, in Proceedings, 4th International Conference on New Photo-Detectors (PhotoDet 2015): Moscow, Russia, July 6-9, 2015, PoS (PhotoDet2015) 038 (2016).

[16] N. Cartiglia, A. Staiano, V. Sola, R. Arcidiacono, R. Cirio, F. Cenna et al., Beam test results of a 16ps timing system based on ultra-fast silicon detectors, NIM A850 (2017) 83-88.

[17] J. Lange, M. Carulla, E. Cavallaro, L. Chytka, P. Davis, D. Flores et al., Gain and time resolution of $45 \mu \mathrm{m}$ thin Low Gain Avalanche Detectors before and after irradiation up to a fluence of $10^{15}$ $n_{\mathrm{eq}} / \mathrm{cm}^{2}$, JINST 12 (2017) P05003. 